\title{
CO Detection and Millimeter Continuum Emission from Low Surface Brightness Galaxies
}

\author{
Mousumi Das ${ }^{1,2}$, Karen O’Neil ${ }^{3}$, Stuart N. Vogel ${ }^{2}$, Stacy McGaugh ${ }^{2}$ \\ ${ }^{1}$ Raman Research Institute, Sadashivanagar, Bangalore, India \\ ${ }^{2}$ Department of Astronomy, University of Maryland, College Park, MD 20742 \\ ${ }^{3}$ NRAO, PO Box 2, Green Bank, WV 24944 \\ email : mousumi@rri.res.in
}

\begin{abstract}
We present BIMA and IRAM $\mathrm{CO}(1-0)$ observations of seven low surface brightness (LSB) galaxies, including three large spirals with faint disks but prominent bulges, and four relatively small LSB galaxies with irregular disks. The giant LSB galaxies are UGC 5709, UGC 6614 and F568-6 (Malin2). The smaller LSB galaxies are NGC 5585, UGC 4115, UGC 5209 and F583-1. The galaxies were selected based on their relatively high metallicity and apparent signs of star formation in their disks. The BIMA maps suggested the presence of molecular gas in 2 of the giant LSB galaxies, F568-6 and UGC 6614. Using the 30m IRAM telescope we detected $\mathrm{CO}(1-0)$ emission in the disks of both galaxies and in the nucleus of F568-6. The molecular gas in these galaxies is clearly offset from the nucleus and definitely associated with the LSB disk. In addition we also detected a millimeter continuum source in the center of UGC 6614. When compared with VLA $1.5 \mathrm{GHz}$ observations of the galaxy, the emission was found to have a flat spectrum indicating that the millimeter continuum emission is most likely due to an active galactic nucleus (AGN) in the galaxy. Our results show that giant LSB spirals may contain significant quantities of molecular gas in their disks and also harbor radio bright AGN in their centers.
\end{abstract}

Subject headings: galaxies:LSB — galaxies:individual (UGC 6614, F568-6) galaxies:ISM — interstellar:molecules — interstellar:kinematics and dynamics — radio lines:galaxies 


\section{INTRODUCTION}

Low surface brightness (LSB) galaxies have faint stellar disks of central brightness less than $23 \mathrm{~B}$ mag $\operatorname{arcsec}^{-2}$ (Impey \& Bothun 1997). Although they have diffuse stellar disks they are extremely gas rich with large HI gas disks that extend well beyond their apparent stellar disks. The high gas fraction observed in these galaxies combined with the overall low metallicity suggests that they have had much less star formation compared to regular high surface brightness (HSB) galaxies, probably because their gas surface densities are well below the critical value required for star formation (van der Hulst et al. 1993). They are thus considered to be less evolved compared to HSB galaxies. They also have very large mass to light ratios which indicate that their disks are dominated by massive dark matter halos; the massive halo inhibits the formation of disk instabilities such as bars and spiral perturbations, which further contributes to the overall low star formation rate in these galaxies.

LSB galaxies span a wide range of Hubble type and size but can be roughly divided into two categories. The first category is made up of the very populous dwarf spiral and dwarf irregular galaxies. The majority of LSB galaxies in our local universe are of this type. The second category comprises of late type spiral galaxies that are very large in size; some may have prominent bulges and discernable spiral arms. These so called "LSB giants" such as Malin 1 and UGC 6614 are relatively rare. The origin and evolution of these faint galaxies is still not clear. Previous studies suggest that LSB galaxies are relatively isolated compared to other types of galaxies and the lack of galaxy interaction has left them less evolved compared to their HSB counterparts. To obtain a better understanding of their evolution, we need to

understand their star formation history. An important step in this direction is to determine the gas distribution in their disks.

Although LSB galaxies are rich in HI, molecular gas $\left(\mathrm{H}_{2}\right)$ has been detected in only a handful of such galaxies (O'Neil, Hofner \& Schinnerer 2000; Matthews \& Gao 2001; O'Neil, Schinnerer \& Hofner 2003; O'Neil \& Schinnerer 2004; Matthews et al. 2005). The low detection rate is probably a combination of several factors such as a low dust content, low metallicity and a low surface density of neutral gas (de Blok \& van der Hulst 1998) all of which impede gas cooling, molecule formation and cloud condensation. However, modest amounts of ongoing star formation have been observed in most late type galaxies (McGaugh \& Bothun 1994; McGaugh, Schombert \& Bothun 1995; Matthews \& Gallagher 1997) which must mean that star formation can proceed even in these adverse environments. Very little is known about the physics of star formation in such low density and low metallicity environments. One possibility is that star formation occurs in localized regions over the disk. Molecular gas is then patchy in distribution and hence difficult to detect.

To address some of these issues we searched for molecular gas in seven LSB galaxies. 
Three of the galaxies are LSB giants and the remaining four are smaller late type galaxies. The parameters of the galaxies are listed in Table 1. All the galaxies in our sample have relatively high metallicities and show signs of star formation in their disks. We followed up tentative BIMA detections of CO (1-0) emission with IRAM 30m single dish observations. CO (1-0) emission was detected in two galaxies, F568-6 (Malin 2) and UGC 6614. The molecular gas masses were derived from the IRAM fluxes; they represent gas masses for the detected regions only and not for the entire galaxy. We also detected millimeter continuum emission from the nucleus of one galaxy in our sample, UGC 6614. The emission was detected at a mean frequency of $111.2 \mathrm{GHz}$ and is most likely due to an AGN in the galaxy.

In the following sections we present our observations and discuss the implications of our results. For F568-6 (Malin 2) which has a fairly high redshift $(\mathrm{z}=0.046$ ), we adopt a luminosity distance of $D_{L}=198.8 \mathrm{Mpc}\left(H_{o}=71 \mathrm{kms}^{-1} \mathrm{Mpc}^{-1}\right)$; this leads to a scale of

$0.92 \mathrm{kpc} \mathrm{arcsec}^{-1}$. UGC 6614 has a much lower redshift and so we adopt the angular distance of $D=89.5 \mathrm{Mpc}\left(V_{\text {sys }}=6351 \mathrm{~km} \mathrm{~s}^{-1}\right)$, which leads to a distance scale of $0.43 \mathrm{kpc} \operatorname{arcsec}^{-1}$.

\section{OBSERVATIONS}

\subsection{GALAXY SAMPLE}

Our sample of seven galaxies is listed in Table 1. To cover the range of galaxy types, we included in our sample both the large LSB spirals and the smaller LSB galaxies that have irregular disks. Also, to improve the chances of molecular gas detection, we chose galaxies that had either relatively high metallicities or showed signs of star formation. Three of the galaxies in the sample, UGC 5709, UGC 6614 and F568-6, are giant LSB galaxies. They are large, late type spirals with diffuse stellar disks but extended HI gas disks. Of these three galaxies, UGC 6614 and F568-6 represent the prototypical giant LSB galaxies (Quillen \& Pickering 1997). Both are relatively bright and nearly face on, have prominent bulges and relatively strong spiral arms (McGaugh, Schombert \& Bothun 1995). The HI gas distribution and kinematics for both galaxies have been observed by Pickering et al. (1997). UGC 6614 has a ring like feature around the bulge (Figure 1) which is prominent even in $H \alpha$ (McGaugh et al. 1995). It has perhaps the highest metallicity known for an LSB galaxy and is estimated as $\log (\mathrm{O} / \mathrm{H})=-3$ to -2.84 , which is close to solar in value (McGaugh 1994). Its nucleus shows AGN activity at optical wavelengths (Schombert 1998) and appears as a bright core in X-ray emission (XMM archival data). F568-6, like UGC 6614, has a massive bulge and spiral structure; in addition it has several bright HII regions distributed over its inner disk. It also has a relatively high metallicity of $\log (\mathrm{O} / \mathrm{H})=-3.22$ which is basically solar in value (de Blok \& van der Hulst 1998). The HI velocity field has high velocities at 
one position near the center which is associated with star formaion and may be due to the accretion of a dwarf (Pickering et al. 1997). This galaxy also shows signs of AGN activity at optical wavelengths (Schombert 1998) and its core has been detected in X-ray emission (XMM archival data). UGC 5709 has a less regular structure than the former 2 galaxies and is intermediate in surface brightness. Its metallicity is $\log (\mathrm{O} / \mathrm{H})=-3$ to -3.2 which is approximately solar (de Blok \& van der Hulst 1998). Like F568-6 it has HII regions in the disk which have relatively high metallicities (McGaugh et al. 1995).

The other 4 galaxies are either dwarf galaxies or irregular galaxies that also have relatively high metallicities or signs of star formation in their disks. They are NGC 5585, UGC 4115, UGC 5209 and F583-1. Apart from F583-1, all the other galaxies are members of groups of galaxies. NGC 5585 is a companion to the bright barred galaxy M101 and has HII regions distributed over the disk. Both UGC 4115 and UGC 5209 are members of loose groups of dwarf galaxies and have irregular morphologies. F583-1 is the only distant galaxy in our sample of dwarf galaxies. It has a metallicity of $\log (\mathrm{O} / \mathrm{H}) \sim-4$ which is substantially subsolar (de Blok \& van der Hulst 1998). It also has a fairly symmetric HI morphology (de Blok, McGaugh \& van der Hulst 1996).

\subsection{BIMA OBSERVATIONS}

We observed the sample of galaxies with the BIMA interferometer (Welch et al. 1996) in the D array from September 2002 to August 2003. The observations were all done in single

pointing mode with a $100^{\prime \prime}$ field of view centered on the galaxy nucleus. The galaxies were observed in the $\mathrm{CO}(1-0)$ emission line, with the line positioned in the upper side band for all 7 galaxies. UGC 5709, UGC 6614 and F568-6 have fairly significant redshifts; the remaining 4 galaxies have low redshifts. We used nearby radio sources for phase calibration; these are listed in Table 2. For flux calibration we used a nearby planet (e.g. Mars) or a radio source whose flux is frequently monitored (3C 273). The data were reduced using MIRIAD (Sault, Teuben \& Wright 1995). We used CLEAN and natural weighting for all our maps. The typical beam size for our maps was $15^{\prime \prime}$ ( see Table 2 for details).

We searched through the channel maps of each galaxy in our sample for signs of CO (1-0) line emission (with velocity resolution of $\sim 6 \mathrm{~km} \mathrm{~s}^{-1}$ ). We also constructed the velocityintegrated CO (1-0) emission map by taking the zeroth moment of the CO (1-0) emission. Our data suggest that two galaxies, UGC 6614 and F568-6, have CO (1-0) emission originating from their disks. However, the emission lines for both galaxies were narrow and noisy. Thus these observations were only an indication that molecular gas may be present in these galaxies. We did not see any CO (1-0) emission in the remaining five galaxies. However, our 
non-detections put upper limits for the CO (1-0) flux one could expect from these galaxies.

In addition to searching for CO (1-0) line emission in the BIMA data, we looked for millimeter continuum emission from all seven galaxies in the sample. To do this we flagged channels that might contain CO (1-0) line emission; the data flagging is based on the velocity distribution of HI emission. We then recomputed the continuum channels from the narrow band dataset. Then we averaged the upper and lower side band data to obtain the combined dataset. We again used CLEAN and natural weighting for deriving the continuum emission maps.

\subsection{IRAM CO OBSERVATIONS}

To confirm the possible detections by BIMA of molecular gas $\left(H_{2}\right)$ in these two galaxies we observed them with the IRAM $30 \mathrm{~m}$ single dish telescope at positions close to the BIMA tentative detections. UGC 6614 and F568-6 were observed in the CO (1-0) and (2-1) emission lines using the IRAM 30m telescope in October-November of 2004 as part of the IRAM $30 \mathrm{~m}$ Pool Observations. Table 3 lists the adopted positions, the frequencies and the total time each position was observed. The beams were centered at the positions given in the table and are $22^{\prime \prime}$ at $110 \mathrm{GHz}$ and $11^{\prime \prime}$ at $220 \mathrm{GHz}$. The positions for IRAM pointing were chosen based on the tentative BIMA CO (1-0) line detections described in Section 2.2. Pointing and focus were checked every 2-3 hours, depending on the weather conditions and pointing was found to be within the telescope limits (better than $2^{\prime \prime}$ ). For each source both transitions were observed simultaneously with both frequencies and two (circular) polarizations. The backend was set using the $1 \mathrm{MHz}$ filterbank for each polarization for the $1 \mathrm{~mm}$ lines and the $4 \mathrm{MHz}$ filterbank for each of the $3 \mathrm{~mm}$ lines. The observed resolution of the $1 \mathrm{~mm}$ and $3 \mathrm{~mm}$ lines were $3.3 \mathrm{~km} \mathrm{~s}^{-1}$ and $5.3 \mathrm{~km} \mathrm{~s}^{-1}$, respectively.

For data reduction, the lines were smoothed over seven channels to $23 \mathrm{~km} \mathrm{~s}^{-1}$ and over five channels to $27 \mathrm{~km} \mathrm{~s}^{-1}$ resolution, for the $\mathrm{J}(1-0)$ and $\mathrm{J}(2-1)$ lines, respectively, using the boxcar smoothing algorithm. The maximal beam throw of $240^{\prime \prime}$ was used for these observations. The image side band rejection ratios were measured to be $>30 \mathrm{~dB}$ for the $3 \mathrm{~mm}$ SIS receivers and $>12 \mathrm{~dB}$ for the $1.3 \mathrm{~mm}$ SIS receivers. The data were calibrated using the standard chopper wheel technique and are reported in main beam brightness temperature $\mathrm{T}_{M B}$. Typical system temperatures during the observations were $160-180 \mathrm{~K}$ and $170-250 \mathrm{~K}$ in the $3 \mathrm{~mm}$ and $1.3 \mathrm{~mm}$ band, respectively. All data reduction was done using CLASS the Continuum and Line Analysis Single-dish Software developed by the Observatoire de Grenoble and IRAM (Buisson 2002). 


\section{RESULTS}

\subsection{CO (1-0) Emission from UGC 6614 and F568-6}

Our BIMA observations suggested the presence of molecular gas at one location in the disk of UGC 6614 (Figures 1 \& 2) and at two positions in the disk of F568-6 (Figure 3, 4 and 5). As described above we followed up these observations with IRAM single dish observations centered near the BIMA tentative detections. We detected CO (1-0) emission from the inner disk of both UGC 6614 and F568-6. We did not detect any emission from the remaining 5 galaxies; however, the BIMA maps yield upper limits to the expected molecular gas masses in these galaxies. In the following paragraphs we discuss the results for each galaxy in more detail.

UGC 6614 : BIMA observations of UGC 6614 indicated that there may be CO (1-0) line emission originating from the disk of UGC 6614 at approximately $38^{\prime \prime}$ west of the nucleus (Figure 1). The emission is weak and so the intensity map does not reveal much about the molecular gas distribution; hence we did not overlay it on the optical image of the galaxy shown in Figure 1. However, though the line is weak $(\mathrm{S} / \mathrm{N} \sim 3.6)$ (Figure 2a) the velocity of the line is similar to the HI line velocity at that point. In the HI map of Pickering et al. (1997) the HI velocity is between 6400 to $6420 \mathrm{~km} \mathrm{~s}^{-1}$; this matches well with the BIMA line. Figure 1 also shows the IRAM pointing center which is offset from the galaxy center by $38^{\prime \prime}$ and the IRAM beam which has a FWHM size of $22^{\prime \prime}$. The IRAM observations detected a significant amount of CO (1-0) flux (Figure 2b); no emission in the CO (2-1) line was detected. The emission is clearly offset from the galaxy center. Thus the BIMA observations suggest that there may be significant CO (1-0) emission from the LSB disk, west of the nucleus and near the spiral arm/ring about the bulge. There may be molecular gas in the nucleus but it is too weak to be detected with BIMA. The molecular gas in the disk appears to be associated with a spiral arm and extended along it. The IRAM observations confirm the presence of gas in the disk. The CO emission may be associated with star formation knots in the inner ring. To derive the molecular gas mass from the single dish CO flux, we used the standard conversion factor which gives the molecular mass as $M_{m o l}=1.5 \times 10^{4} D_{M p c}{ }^{2} S_{C O} \mathrm{M}_{\odot}$, where $D_{M p c}$ is the galaxy distance in Mpc and $S_{C O}$ is the velocity integrated flux in Jy km s${ }^{-1}$ (Strong et al. 1988; Scoville et al. 1987). The total molecular gas mass observed in the disk from this region is $\sim 2.8 \times 10^{8} M_{\odot}$ (Table 4 ).

F568-6 : BIMA observations of F568-6 suggested that there may be CO (1-0) emission originating from 2 positions in the inner disk of the galaxy. One position was close to the nucleus (F568-6B) and the other was clearly offset from the nucleus and close to a spiral feature in the disk (F568-6A). Although the emission lines seen in the BIMA data were 
relatively weak, the velocities of the lines match observed HI velocities at both positions and hence appear to be real. Figure 3 shows the BIMA CO intensity map superimposed on the $\mathrm{R}$ band image of the galaxy. The IRAM pointings were at two locations in the disk and are marked as $\mathrm{A}$ and $\mathrm{B}$ in the figure; they are offset by $\sim 35^{\prime \prime}$ and $\sim 7^{\prime \prime}$ respectively from the galaxy center. Also shown is the IRAM beam that has a FWHM of $22^{\prime \prime}$.

The IRAM $\mathrm{CO}(1-0)$ emission from position A (Figure 4) has a line velocity of approximately $13990 \mathrm{~km} \mathrm{~s}^{-1}$. This matches the HI velocity observed at that position by Pickering et al. (1997) which lies between 13980 to $14020 \mathrm{~km} \mathrm{~s}^{-1}$. The corresponding BIMA line (Figure 4a) is at a velocity of $\sim 14010 \mathrm{~km} \mathrm{~s}^{-1}$ which is slightly offset from the center of the IRAM line (Figure $4 \mathrm{~b}$ ). This may be because the molecular gas is distributed as clumps over the beam rather than a large gas cloud. Our BIMA observations are thus picking up emission from one bright spot whereas the IRAM beam is sensitive to emission from the entire cloud complex. The emission from A is clearly positioned away from the galaxy nucleus; it lies to the east of the galaxy center and appears to be associated with a tightly wound spiral arm emerging from the edge of the bulge. The IRAM $\mathrm{CO}(1-0)$ emission from position $\mathrm{B}$ has a peak velocity of approximately $13910 \mathrm{~km} \mathrm{~s}^{-1}$ (Figure 5) and the HI velocity at that position is between 13900 to $13920 \mathrm{~km} \mathrm{~s}^{-1}$ (Pickering et al. 1997). This emission appears to be associated with the nuleus. For this position, the BIMA and IRAM line velocities are fairly well matched (Figure 5a and b).

To derive the molecular gas masses we used the CO luminosity $L_{C O}$ ' i.e. $M_{\text {mol }}=$ $\alpha \times L_{C O}{ }^{\prime} \mathrm{M}_{\odot}$, where $\alpha=4 M_{\odot}\left(K \mathrm{~km} \mathrm{~s}^{-1} \mathrm{pc}^{2}\right)^{-1}$ (Strong et al. 1988; Scoville et al. 1987). The total molecular gas mass derived from summing up the gas detected at both positions A and B using the IRAM CO (1-0) flux is $\sim 2.6 \times 10^{9} M_{\odot}$ (Table 4). The emission is extended east of the galaxy center but the molecular gas may well be extended west of the nucleus as well (Figure 3). This is because the correlator setup for this galaxy did not cover the full range of velocities for which $\mathrm{HI}$ emission has been detected in this galaxy, since the lower edge of the correlator band was set at $13,860 \mathrm{~km} \mathrm{~s}^{-1}$. Thus our present detection is only a lower limit to the molecular gas distribution in F568-6 and is based only on the redshifted half of the galaxy. Hence the molecular gas may be much more extended in the inner disk of the galaxy than our present observations indicate. In the near future we plan to map the extended molecular gas distribution in this galaxy in much better detail.

Remaining Galaxies : We did not detect any CO (1-0) emission from the remaining five galaxies in our sample (i.e. UGC 5709, NGC 5585, UGC 4115, UGC 5209 and F5831). Of these galaxies one is a giant LSB galaxy (UGC 5709) and the others are dwarf galaxies. However we have used the noise levels in the BIMA maps to derive upper estimates of the molecular gas masses in these galaxies (Table 4) using the approximation $S_{C O}=$ 
(noise) $\times(\delta v) \times\left(N_{b}\right)$ where $\delta v$ represents the maximum width of the expected $\mathrm{CO}(1-0)$

emission line; $N_{b}$ is number of beams in the $44^{\prime \prime}$ BIMA primary beam (i.e. $\frac{44^{\prime \prime}{ }^{2}}{b_{1} \times b_{2}}$ ) and $b_{1}, b_{2}$ are the beam sizes. For $\delta v$ we have used the width of the HI line $\left(W_{50}\right)$ as an upper estimate. Upper limits to the molecular gas masses is calculated from the flux using the previously mentioned formula $M_{m o l}=1.5 \times 10^{4} D_{M p c}{ }^{2} S_{C O} \mathrm{M}_{\odot}$ (Table 4).

\section{2. $3 \mathrm{~mm}$ Continuum Emission from UGC 6614}

We searched for $3 \mathrm{~mm}$ continuum emission from all the galaxies in our sample. Of the seven galaxies that we observed two have been detected in radio continuum at $1.4 \mathrm{GHz}$ in the NRAO VLA Sky Survey (NVSS) (Condon et al. 1998); they are UGC 6614 and F568-6. Both galaxies are also strong emitters in the VLA FIRST Survey (Becker, White \& Helfand 1995) which is at a similar frequency but has a much higher resolution than NVSS $\left(\sim 5^{\prime \prime}\right)$.

We detected only UGC 6614 in 3mm continuum emission using BIMA (Figure 6); the remaining 6 galaxies were not detected. Table 5 lists the continuum $3 \mathrm{~mm}$ flux observed in UGC 6614 and upper limits for the remaining galaxies. In UGC 6614 the continuum emission is detected at a mean frequency of $111.2 \mathrm{GHz}$ and the peak flux at this frequency is $4.9 \mathrm{mJy} /$ beam. The noise in the map is $1.2 \mathrm{mJy} /$ beam which means that the detection has a signal to noise ratio $(\mathrm{S} / \mathrm{N})$ of approximately 4 . The beam is $\sim 16^{\prime \prime}$. The position of the peak in the continuum source is $11^{h} 39^{m} 14^{s} .8,17^{h} 08^{m} 37^{s} .5$; the error is $\pm 4^{\prime \prime}$. Within error limits the continuum emission peak is definitely coincident with the 2MASS location of the galaxy center (Table 1). Although the $\mathrm{S} / \mathrm{N}$ of the detection is not high, the fact that the continuum source is located at the galaxy nucleus raises the significance of the detection.

We calculated the spectral index of the continuum emission for UGC 6614 and upper limits for F568-6 using data from the FIRST 1.4 GHz VLA survey. We convolved the FIRST $1.4 \mathrm{GHz}$ maps of UGC 6614 and F568-6 to the same resolution as the BIMA maps and measured the peak flux. Table 5 lists the spectral index for UGC 6614 and upper limits for F568-6. The spectrum is flat for UGC $6614\left(\alpha \sim 0\right.$ where $\left.f_{\nu} \propto \nu^{\alpha}\right)$ and is likely declining at millimeter wavelengths for F568-6. As both galaxies host AGN and have little star formation activity, the radio continuum emission is probably non-thermal in nature. However, for UGC 6614, we confirmed this by using the $H \alpha$ flux in the galaxy (de Blok \& van der Hulst 1998) to derive the star formation rate (SFR) and infrared luminosity (Kennicutt 1983; Kennicutt 1998). The expected $1.4 \mathrm{GHz}$ radio continuum flux from the $H \alpha$ emission is only $0.15 \mathrm{mJy}$. This is much less than the actual continuum flux measured in the $1.4 \mathrm{GHz}$ map of UGC 6614 which is $5.67 \mathrm{mJy}$. Thus the BIMA 3mm continuum emission from UGC 6614, is non-thermal in nature and due to an AGN in the galaxy. As mentioned 
earlier, AGN activity in UGC 6614 has been detected at optical wavelengths (Schombert 1998) and also in X-ray emission (XMM archive).

\section{DISCUSSION}

We have detected CO (1-0) line emission from the disks of two giant LSB galaxies, F568-6 and UGC 6614. We have also detected and mapped the millimeter continuum source in the nucleus of UGC 6614. In the following paragraphs we discuss the implications of our findings.

\subsection{Molecular Gas Detection}

As mentioned in Section 1, very few LSB galaxies have been detected in CO emission. Of the handful of galaxies in which molecular gas has been detected some are giant, bulge dominated spirals with large disk rotation velocities (O'Neil et al. 2000, 2003) while others are more low mass, disk dominated systems (Matthews \& Gao 2001, Matthews et al. 2005). However, in nearly all previous detections the molecular gas has been found to be centrally concentrated and associated with the galaxy bulge or nucleus. The exception being the single dish observations of Matthews \& Gao (2001) which show CO emission originating from off center positions for two galaxies in their sample. Our detections of $\mathrm{CO}$ emission from UGC 6614 and F568-6 are however unique because both detections have interferometric as well as single dish observations of the molecular gas distribution that clearly indicate that they are associated with the disk and not just the galaxy center. This is important as it shows that molecular gas can form and exist in the disks of LSB galaxies as well as in their nuclear regions.

Previous studies of LSB galaxies suggest that the low detection rate of molecular gas is a result of a combination of low metallicity and low gas surface density in the disk (van der Hulst et al. 1993; de Blok et al. 1996; Mihos, Spaans \& McGaugh 1999). In addition LSB galaxies have massive dark halos that may prevent global disk instabilities from forming even when they interact with other galaxies (Mihos, McGaugh and de Blok 1997); this reduces the formation of shocks that form dense clouds and lead to massive star formation in galaxy disks (Gerritsen \& de Blok 1999). In LSB disks the molecular gas may not form due to such large scale instabilities. Instead it may form due to local instabilities which will result in isolated clumps of molecular gas and localized regions of modest star formation. Signatures of such localized star formation has been observed in LSB galaxies (Schombert et al. 1990; 
McGaugh et al. 1995) and our observations of molecular gas in the disks of both UGC 6614 and F568-6 further support this idea. Thus LSB galaxies may not be as devoid of molecular gas as thought earlier; the distribution may just be localized to isolated regions over the disk and hence difficult to detect.

Another possibilty that is hard to exclude is that given the physical conditions of the ISM in LSB galaxies, the $\mathrm{CO}$ to $\mathrm{H}_{2}$ conversion factor is different from the standard value (Mihos et al. 1999; Gerritsen \& de Blok 1999). This might allow for substantially more molecular gas to be present than we infer. Bearing this caveat in mind, our observations nevertheless seem to suggest a picture in which molecular gas is rare in LSB galaxies but not completely absent. Such a distribution of molecular gas supports the observed, low intensity star formation in isolated regions that is seen in these galaxies.

\subsection{Continuum Emission}

Figure 6 shows the BIMA map of the continuum source in UGC 6614. The central peak coincides with the 2MASS center of the galaxy; the offset between the two is less than an arcsecond. The emission is probably non-thermal in nature and due to an AGN in the galaxy. Several giant LSB galaxies that have prominent bulges have been found to host AGN and show weak Seyfert activity. AGN activity in LSB galaxies has been detected in optical emission lines (Schombert 1998; Sprayberry et al 1995). The nuclear activity is due to the accretion of mass onto a supermassive black hole in the galactic center. It is now widely accepted that most galaxies with a well defined bulge contain a black hole (Magorrian et al. 1998) and that the bulge mass correlates well with the black hole mass (Ferrarese et al. 2001). Giant LSB galaxies have both prominent bulges and copious amounts of neutral hydrogen (HI) gas in their inner disks. Hence it is not suprising that these galaxies show signs of AGN activity. However it is not clear how the gas is transported to the nucleus since gas fueling processes such as bars are not frequently found in these galaxies (Mihos et al. 1997). It could be that since the AGN activity is fairly weak in LSB galaxies, the gas torquing produced by the disk spiral arms is enough to funnel gas into the nuclear regions and fuel the AGN.

Very little is known about the continuum emission from LSB galaxies. A handful appear bright in the NVSS and FIRST surveys which are at $1.4 \mathrm{GHz}$, but none have been studied at millimeter wavelengths (e.g. $100 \mathrm{GHz}$ ). The continuum source at $111 \mathrm{GHz}$ in UGC 6614 is the first such detection at millimeter wavelenghths. Suprisingly F568-6 is not bright at millimeters wavelengths although its core is bright in the $1.4 \mathrm{GHz}$ continuum maps (NVSS, FIRST). This implies that the synchroton spectrum of F568-6 turns over from a flat spectrum 
to a steeply falling spectrum before millimeter wavelengths, whereas in UGC 6614 it remains constant. A similar difference in turnover frequencies is observed in the nearby Seyfert galaxies NGC 1068 and NGC 3147 (Krips et al. 2005) and is probably due to the different magnetic field strengths in their AGN (e.g. Krolik 1999). Flat spectrum radio galaxies also show a variation in turnover frequencies between centimeter to millimeter wavelenghths (Bloom et al. 1999) but not many sources are found to be bright at millimeters. Thus UGC 6614 is unique with respect to its nuclear continuum source which is radio bright even at millimeter wavelengths.

\section{CONCLUSIONS}

We have searched for molecular gas in a sample of seven LSB galaxies using the BIMA interferometer. Molecular gas was detected in the disks of two galaxies, UGC 6614 and F5686 , using the IRAM 30m single dish telescope. Both galaxies have prominent bulges and large, low surface brightness disks. Our results indicate that molecular gas may be present in both the disks of LSB galaxies as well as their nuclei but the distribution is localized over isolated regions and thus difficult to detect in unbiased single dish observations. Overall it appears that molecular gas is rare in these galaxies but not completely absent. We have also detected

millimeter continuum emission from the nucleus of one of these galaxies, UGC 6614. Our main results are summarised below.

1. CO (1-0) emission was detected in UGC 6614 at approximately $17 \mathrm{kpc}\left(38^{\prime \prime}\right)$ west of the nucleus. It is associated with a spiral feature in the disk. The mass of molecular gas detected from this location is $\sim 2.8 \times 10^{8} M_{\odot}$. The BIMA observations did not detect any molecular gas in the nuclear region, probably because the gas surface density averaged over the BIMA beam falls below the detection limits of the observation.

2. CO (1-0) emission in F568-6 was detected at two positions in the inner disk. One position is close to the nucleus and the other is approximately $28 \mathrm{kpc}\left(30^{\prime \prime}\right)$ east of the nucleus. The BIMA intensity maps indicate that the CO (1-0) emission is distributed about the nucleus and extends into the inner disk. The mass of molecular gas associated with the detection is $\sim 2.6 \times 10^{9} M_{\odot}$.

3. We have also detected a millimeter continuum source in UGC 6614 at the center of the galaxy. Comparing it with VLA FIRST $1.5 \mathrm{GHz}$ maps, we find that it has a flat spectrum between 1 to $110 \mathrm{GHz}$. The continuum emission is due to the AGN in the galaxy which has been detected at optical and X-ray wavelengths as well. Millimeter continuum emission from Seyferts or radio loud galaxies is rare and so UGC 6614 is fairly unique in its 
AGN activity compared to most spiral galaxies.

The authors would like to thank Alice Quillen for providing the $\mathrm{R}$ band images of UGC 6614 and F568-6. Observations with the BIMA millimeter-wave array are partially supported by NSF AST-0228974. We are grateful to the members of the IRAM staff for their help in the observations. This research has made use of the NASA/ IPAC Infrared Science Archive, which is operated by the Jet Propulsion Laboratory, California Institute of Technology, under contract with the National Aeronautics and Space Administration. We also acknowledge the usage of the HyperLeda database (http://leda.univ-lyon1.fr). 


\section{REFERENCES}

Becker, R.H.; White, R.L.; Helfand, D.J. 1995, ApJ, 450, 559

Bloom, S.D. et al. 1999, ApJS, 122, 1

Buisson, G. et al. 2002, CLASS Continuum and Line Analysis System Handbook online at http://iram.fr/GS/class/class/html

Condon, J.J.; Cotton, W.D.; Greisen, E.W., Yin, Q.F., Perley, R.A., Taylor, G.B. \& Broderick, J. 1998, AJ, 115, 1693

Cote, S., Carignan, C., Sancisi, R., 1991, AJ, 102, 904

de Blok, W. J. G., McGaugh, S.S. \& van der Hulst, J.M. 1996, MNRAS, 283, 18

de Blok, W. J. G. \& van der Hulst, J. M. 1998, A\&A, 336, 49

de Blok, W. J. G.; McGaugh, Stacy S.; Rubin, Vera C. 2001, AJ, 122, 2396

Ferrarese, L. et al. 2001, ApJ, 555, L79

Gerritsen, J.P.E. \& de Blok, W.J.G. 1999, A\&A, 342, 655

Hoffman, G. Lyle; Salpeter, E. E.; Farhat, B.; Roos, T.; Williams, H.; Helou, G. 1996, ApJS, 105,269

Impey, C. \& Bothun, G. 1997, ARA\&A, 35, 267

Karachentsev, I.D., Karachentseva, V.E., Huchtmeier, W.K. 2001, A\&A, 366, 428

Kennicutt, R.C. 1983, ApJ, 272, 54

Kennicutt, R.C. 1998, ARA\&A, 36, 189

Krips, M. et al. 2005, astro-ph/0509825

Krolik, J.H. 1999, Active Galactic Nuclei, Princeton University Press

Magorrian, J. et al. 1998, AJ, 115, 2285

Matthews, L.D. \& Gallagher, J.S. 1997, AJ, 114, 1899

Matthews, L.D. \& Gao, Y. 2001, ApJ 549, L191

Matthews, L.D.; Gao, Y.; Uson, J.M.; Combes, F. 2005, AJ, 129, 1849

McGaugh, S.S. 1994, ApJ, 426, 135

McGaugh, S.S. \& Bothun, G.D. 1994; AJ, 107, 530

McGaugh, S.S.; Schombert, J.M. \& Bothun, G.D. 1995, AJ, 109, 2019

Mihos, J.C.; McGaugh, S.S. \& de Blok, W. J. G. 1997, ApJL, 477, L79

Mihos, J.C.; Spaans, M. \& McGaugh, S.S. 1999, ApJ, 515, 89 
O’Neil, K.; Hofner, P. \& Schinnerer, E. 2000, ApJ, 545, L102

O’Neil, K.; Schinnerer, E.; \& Hofner, P. 2003, ApJ, 588, 230

O’Neil, K. \& Schinnerer, E. 2004, ApJL 615, 109

Quillen, A. C.; Pickering, T. E. 1997, AJ, 113, 2075

Paturel, G.; Theureau, G.; Bottinelli, L.; Gouguenheim, L.; Coudreau-Durand, N.; Hallet, N.; Petit, C. 2003, A\&A, 412, 45

Pickering, T. E.; Impey, C. D.; van Gorkom, J. H.; Bothun, G. D. 1997, AJ, 114, 1858

Sault, R. J.; Teuben, P. J.; Wright, M. C. H. 1995, ADASS, ASP Conference Series, Vol. 77, eds. R.A. Shaw, H.E. Payne, and J.J.E. Hayes, p. 433.

Schombert, J.M.; Bothun, G.D.; Impey, C. D. \& Mundy, L.G. 1990, AJ, 100, 1523

Schombert, J.M. 1998; AJ, 116, 1650

Scoville, N. Z.; Sanders, D. B. 1987, in Interstellar processes, ed. D. Hollenbach \& H. Thronson (Dordrecht, D. Reidel), p.21

Sprayberry, D; Impey, C.D.; Bothun, G.D. \& Irwin, M.J. 1995, AJ, 109, 558

Strong, A. W. et al. 1988, A\&A, 207, 1

van der Hulst, J.M.; Skillman, E.D.; Smith, T.R.; Bothun, G.D.; McGaugh, S.S. \& de Blok, W.J.G. 1993 AJ, 106, 548

Welch, W.J. et al. 1996, PASP, 108, 93 


\section{FIGURE CAPTIONS}

Figure 1. The R band optical image of UGC 6614; the location of the center of the IRAM beam is indicated with the arrow labeled A. The telescope pointing center is well away from the center of the galaxy. The IRAM beam size is $22^{\prime \prime}$ and the $2 \mathrm{MASS}$ center of the galaxy is marked with a filled triangle.

Figure 2. Panel showing the BIMA and IRAM CO (1-0) emission lines observed in UGC 6614. The topmost panel is a CO spectrum observed by BIMA from the nuclear region; no CO (1-0) emission is seen here. The middle panel shows the BIMA CO (1-0) spectrum observed from location A that lies west of the nucleus and in the LSB disk. Both spectra have been smoothed to a velocity resolution of $6 \mathrm{~km} \mathrm{~s}^{-1}$. The lower panel is the IRAM single dish CO (1-0) spectrum smoothed to a velocity resolution of $27 \mathrm{~km} \mathrm{~s}^{-1}$. The systemic velocity of the galaxy is marked on the $\mathrm{x}$ axis with an arrow. The dashed lines show the approximate width of the single dish emission line.

Figure 3. Contours of BIMA C0 (1-0) line emission overlaid on the R band optical image of F568-6 (Malin 2). The intensity contours in white are at 1.5, 2, 2.5, 3 and 3.5 times the rms noise level and the contours in black are at -1.5 and -2.0 times the $\mathrm{rms}$ noise level. The beam is $\sim 17^{\prime \prime}$. The pointing centers of the $22^{\prime \prime}$ IRAM beams for the two C0 (1-0) emission detections are indicated with the arrows labeled A and B. The 2MASS center of the galaxy is marked with a filled triangle.

Figure 4. Panel showing the BIMA and IRAM CO (1-0) emission lines observed in F568-6 from the location marked A in Figure 3 (i.e. approximately $35^{\prime \prime}$ west of the nucleus). The upper panel shows the BIMA CO (1-0) spectrum smoothed to a velocity resolution of $6 \mathrm{~km} \mathrm{~s}^{-1}$; the lower panel shows the IRAM single dish CO (1-0) spectrum smoothed to a velocity resolution of $27 \mathrm{~km} \mathrm{~s}^{-1}$. The systemic velocity of the galaxy is marked on the $\mathrm{x}$ axis with an arrow. The dashed lines show the approximate width of the single dish emission line.

Figure 5. Panel showing the BIMA and IRAM CO (1-0) emission lines observed in F568-6 from the location marked B in Figure 3 (i.e. close to the nucleus). The upper panel shows the BIMA CO (1-0) spectrum smoothed to a velocity resolution of $6 \mathrm{~km} \mathrm{~s}^{-1}$; the lower panel shows the IRAM single dish CO (1-0) spectrum smoothed to a velocity resolution of $27 \mathrm{~km} \mathrm{~s}$. The systemic velocity of the galaxy is marked on the $\mathrm{x}$ axis with an arrow. The dashed lines show the approximate width of the single dish emission line.

Figure 6. BIMA continuum emission map of UGC 6614 at $2.7 \mathrm{~mm}$. The emission is averaged over the upper and lower sidebands, with a mean frequency of $111.2 \mathrm{Ghz}$. The emission is centered on the 2MASS near-infrared position of the nucleus. The beam is shown on the lower left. The emission is contoured at $-2,-2.5,2,2.5,3,3.5$ and 4 times the noise level. The noise level is $1.2 \mathrm{mJy} /$ beam where the beam is $17.4 \times 15^{\prime \prime}$. The peak emission is $4.9 \mathrm{mJy} /$ beam. 
Table 1. Galaxy Sample

\begin{tabular}{lccccc}
\hline \hline $\begin{array}{c}\text { Galaxy } \\
\text { Name }\end{array}$ & $\begin{array}{c}\text { Galaxy } \\
\text { Type }\end{array}$ & $\begin{array}{c}\text { Velocity } \\
\mathrm{km} \mathrm{s}^{-1}\end{array}$ & $\begin{array}{c}\text { Galaxy Position } \\
\text { RA, } \delta(\mathrm{J} 2000)\end{array}$ & $\begin{array}{c}D_{25} \\
\prime\end{array}$ & $\begin{array}{c}\text { inclination } \\
\text { degrees }\end{array}$ \\
\hline UGC 5709 & Sd & 6206 & $10^{h} 31^{m} 16^{s} .2,+19^{\circ} 22^{\prime} 59^{\prime \prime}$ & 80.9 & 54.6 \\
UGC 6614 & (R)SA(r)a & 6351 & $11^{h} 39^{m} 14^{s} .8,+17^{\circ} 08^{\prime} 37^{\prime \prime}$ & 99.6 & 29.9 \\
F568-6 & Sd/p & 13820 & $10^{h} 39^{m} 52^{s} .5,+20^{\circ} 50^{\prime} 49^{\prime \prime}$ & $\ldots$ & 38.0 \\
NGC 5585 & SAB(s)d & 305 & $14^{h} 19^{m} 48^{s} .2,+56^{\circ} 43^{\prime} 45^{\prime \prime}$ & 345.3 & 53.2 \\
UGC 4115 & IAm & 338 & $07^{h} 57^{m} 01^{s} .8,+14^{\circ} 23^{\prime} 27^{\prime \prime}$ & 109.2 & 67.1 \\
UGC 5209 & Im & 538 & $09^{h} 45^{m} 04^{s} .2,+32^{\circ} 14^{\prime} 18^{\prime \prime}$ & 54.7 & 0.0 \\
F583-1 & Sm/Irr & 2264 & $15^{h} 57^{m} 27^{s} .5,+20^{\circ} 39^{\prime} 58^{\prime \prime}$ & 42.0 & 63.0 \\
\hline
\end{tabular}

(a) The $D_{25}$ diameters for all the galaxies is from the RC3 catalogue, except for F583-1 which is from de Blok, McGaugh \& van der Hulst (1997).

(b) The inclinations for all the galaxies except F568-6 and F583-1 is from Hyperleda catalogue (Paturel et al. 2003). For F568-6, the inclination is from Pickering et al. (1997) and for F583-1 the inclination is obtained from de Blok, McGaugh \& van der Hulst (1997).

Table 2. BIMA Observations

\begin{tabular}{lccccc}
\hline \hline $\begin{array}{c}\text { Galaxy } \\
\text { Name }\end{array}$ & $\begin{array}{c}\text { CO(1-0) } \\
\text { Frequency }(\mathrm{GHz})\end{array}$ & $\begin{array}{c}\text { Phase } \\
\text { Calibrator }\end{array}$ & $\begin{array}{c}\text { Flux } \\
\text { Calibrator }\end{array}$ & $\begin{array}{c}\text { Beam } \\
\text { Size }\end{array}$ & $\begin{array}{c}\text { Number } \\
\text { of tracks }\end{array}$ \\
\hline UGC 5709 & 112.93 & $1058+015$ & $3 \mathrm{C} 273$ & $18.5^{\prime \prime} \times 14.5^{\prime \prime}$ & 3 \\
UGC 6614 & 112.88 & $1118+125$ & Mars & $17.3^{\prime \prime} \times 15.0^{\prime \prime}$ & 3 \\
F568-6 & 110.19 & $1058+015$ & 3C 273 & $18.4^{\prime \prime} \times 16.5^{\prime \prime}$ & 6 \\
NGC 5585 & 115.15 & $1419+543$ & Mars & $17.3^{\prime \prime} \times 13.9^{\prime \prime}$ & 3 \\
UGC 4115 & 115.14 & $0750+125$ & Mars & $17.3^{\prime \prime} \times 14.6^{\prime \prime}$ & 1 \\
UGC 5209 & 115.07 & $0927+390$ & W3OH & $16.9^{\prime \prime} \times 13.8^{\prime \prime}$ & 2 \\
F583-1 & 114.41 & $1540+147$ & Mars & $16.4^{\prime \prime} \times 15.7^{\prime \prime}$ & 1 \\
\hline
\end{tabular}


Table 3. IRAM Observations

\begin{tabular}{lccccc}
\hline \hline $\begin{array}{c}\text { Galaxy } \\
\text { Name }\end{array}$ & RA & DEC & $\begin{array}{c}\mathrm{CO}(1-0) \\
\text { Frequency (GHz) }\end{array}$ & $\begin{array}{c}\mathrm{CO}(2-1) \\
\text { Frequency (GHz) }\end{array}$ & $\begin{array}{c}\text { Time } \\
\text { in mins }\end{array}$ \\
\hline F568-6a & $10^{h} 39^{m} 55^{s}$ & $20^{h} 50^{m} 54^{s}$ & 110.20559 & 220.40696 & 134 \\
F568-6b & $10^{h} 39^{m} 53^{s}$ & $20^{h} 50^{m} 50^{s}$ & 110.31817 & 220.63212 & 240 \\
UGC 6614 & $11^{h} 39^{m} 12^{s}$ & $17^{h} 08^{m} 31^{s}$ & 112.89500 & 225.78569 & 184 \\
\hline
\end{tabular}

Table 4. CO Fluxes and Molecular Gas Masses

\begin{tabular}{|c|c|c|c|c|c|c|c|}
\hline $\begin{array}{l}\text { Galaxy } \\
\text { Name }\end{array}$ & $\begin{array}{l}\text { BIMA Peak } \\
\text { Flux } \\
\left(\text { Jy beam }^{-1}\right)\end{array}$ & Noise & $\begin{array}{l}\text { Channel } \\
\text { Width } \\
\left(\mathrm{km} \mathrm{s}^{-1}\right)\end{array}$ & $\begin{array}{l}\text { HI Width } \\
\text { W }_{50} \\
\left(\mathrm{~km} \mathrm{~s}^{-1}\right)\end{array}$ & $\begin{array}{c}\text { IRAM } \\
\text { Flux } \\
\left(\mathrm{Jy} \mathrm{km} \mathrm{s}^{-1}\right)\end{array}$ & Noise & $\begin{array}{c}\text { Molecular } \\
\text { Gas Mass }^{a} \\
\quad\left(\mathrm{M}_{\odot}\right)\end{array}$ \\
\hline UGC 6614 & 0.060 & 0.017 & 6.1 & 242 & 2.30 & 0.006 & $2.8 \times 10^{8}$ \\
\hline F568-6 A & 0.043 & 0.013 & 6.1 & $\ldots$ & 1.86 & 0.005 & $0.7 \times 10^{9}$ \\
\hline F568-6 B & 0.057 & 0.013 & 6.1 & $\ldots$ & 5.24 & 0.005 & $1.9 \times 10^{9}$ \\
\hline UGC 4115 & $\ldots$ & 0.047 & 6.1 & 83 & $\ldots$ & $\ldots$ & $<1.1 \times 10^{7}$ \\
\hline UGC 5209 & $\ldots$ & 0.007 & 6.1 & 49 & $\ldots$ & $\ldots$ & $<2.4 \times 10^{6}$ \\
\hline UGC 5585 & $\ldots$ & 0.039 & 6.1 & 146 & $\ldots$ & $\ldots$ & $<1.3 \times 10^{7}$ \\
\hline UGC 5709 & $\ldots$ & 0.022 & 6.1 & 247 & $\ldots$ & $\ldots$ & $<4.6 \times 10^{9}$ \\
\hline F583-1 & $\ldots$ & 0.056 & 6.1 & 174 & $\ldots$ & $\ldots$ & $<1.1 \times 10^{9}$ \\
\hline
\end{tabular}

(a) Molecular gas masses were derived from IRAM fluxes; the values represent gas masses for the detected regions only and not for the entire galaxy. The upper limits for the non-detections in BIMA were derived using the noise levels in the corresponding channel maps and the width of the HI line $\left(W_{50}\right)$. For F583-1, twice the HI rotation curve peak was used (de Blok, McGaugh \& Rubin 2001) for width of the HI line instead of $W_{50}$. 
Table 5. Continuum Fluxes and Spectral Indices

\begin{tabular}{lccccc}
\hline \hline $\begin{array}{c}\text { Galaxy } \\
\text { Name }\end{array}$ & $\begin{array}{c}\text { BIMA Continuum } \\
\text { Flux (mJy/b) }\end{array}$ & $\begin{array}{c}\text { Frequency } \\
\text { GHz }\end{array}$ & $\begin{array}{c}\text { FIRST Continuum } \\
\text { Flux }(\mathrm{mJy} / \mathrm{b})\end{array}$ & $\begin{array}{c}\text { Frequency } \\
\text { GHz }\end{array}$ & Spectral Index \\
\hline UGC 6614 & 4.9 & 111.25 & 5.5 & 1.4 & -0.03 \\
F568-6 & $<2.4$ & 108.4 & 5.4 & 1.4 & $<-0.19$ \\
\hline UGC 4115 & $<8.4$ & 113.5 &.. &.. &.. \\
UGC 5209 & $<1.2$ & 113.5 &.. &.. &.. \\
NGC 5585 & $<6.3$ & 113.5 &.. &.. &.. \\
UGC 5709 & $<4.5$ & 111.3 &.. &.. &.. \\
F583-1 & $<10.5$ & 112.8 &.. &.. &.. \\
\hline
\end{tabular}

(a) The upper limits for the non-detections of BIMA continuum fluxes are given as three times the noise levels in the corresponding maps.

(b) The FIRST map is smoothed to the same resolution of the BIMA map before estimating the continuum flux. 


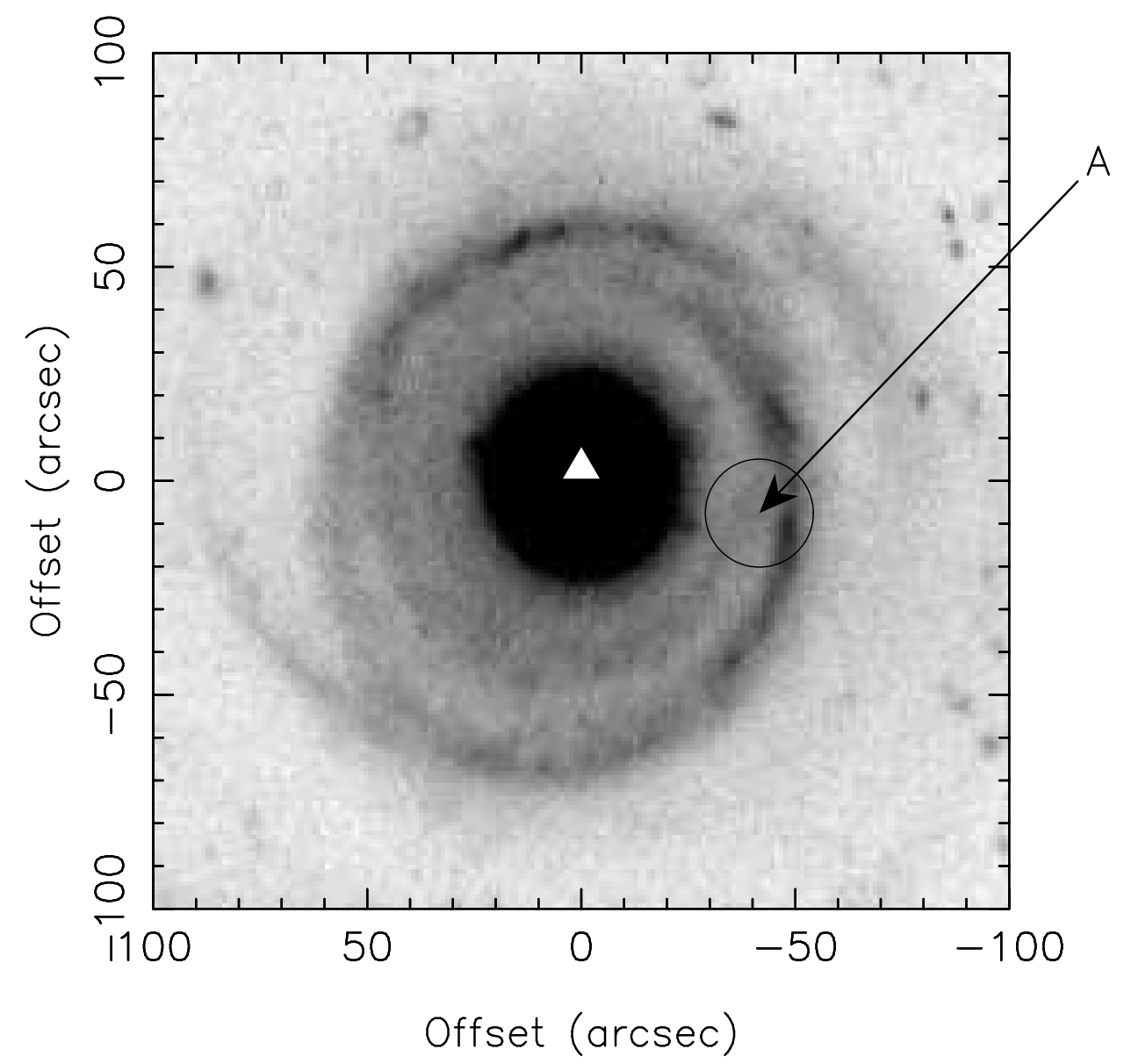




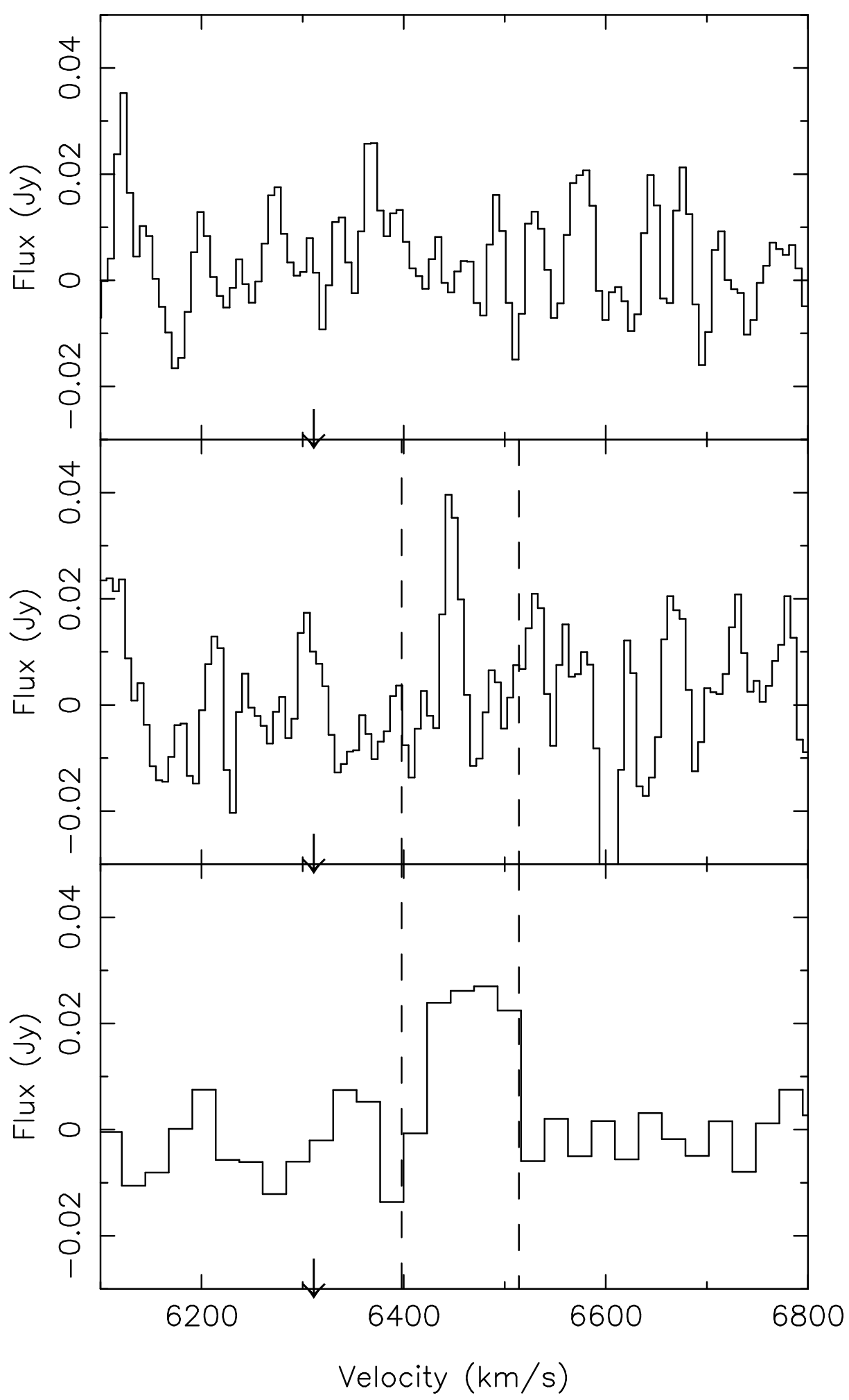


This figure "f3.gif" is available in "gif" format from: http://arxiv.org/ps/astro-ph/0606746v1 

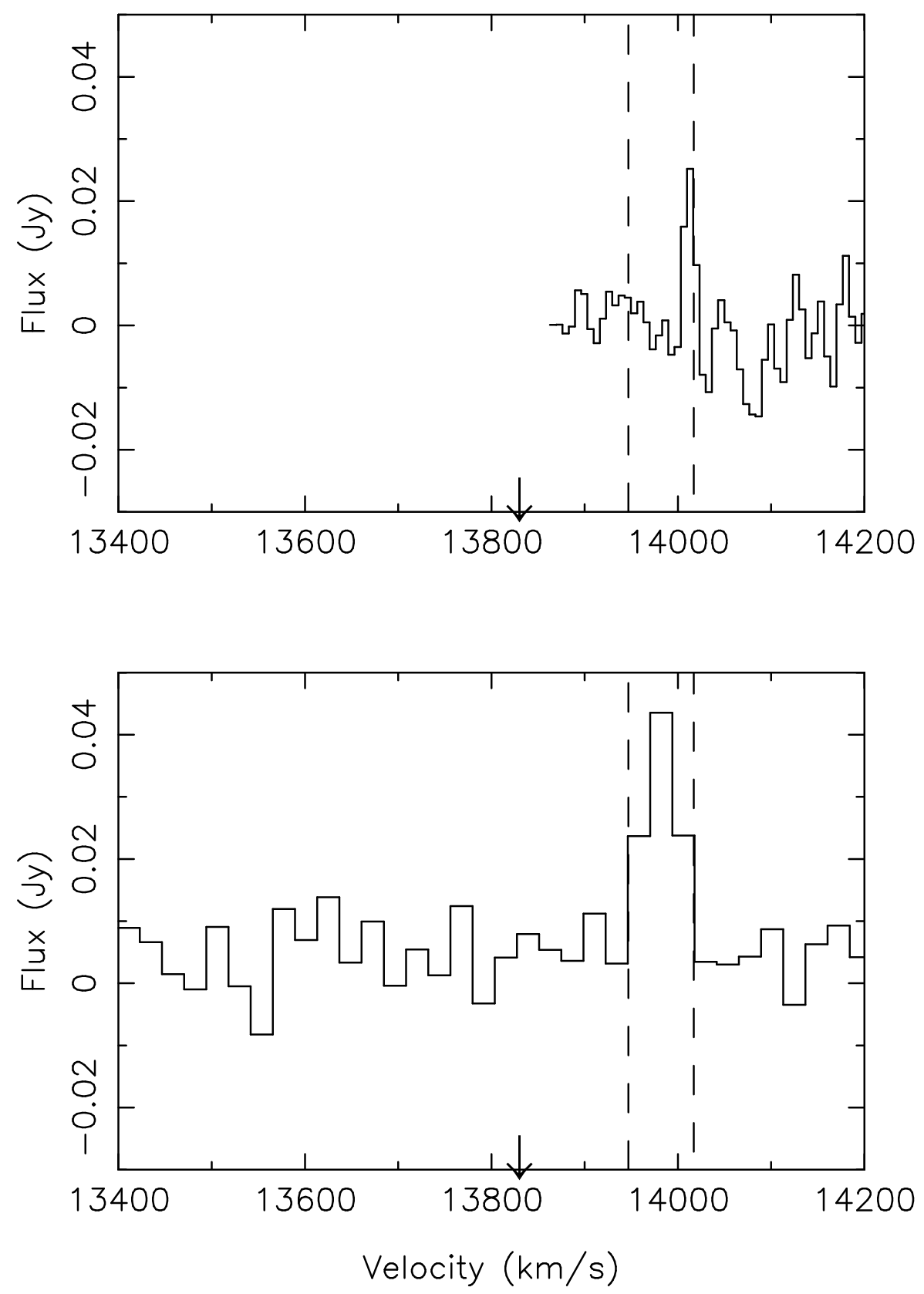

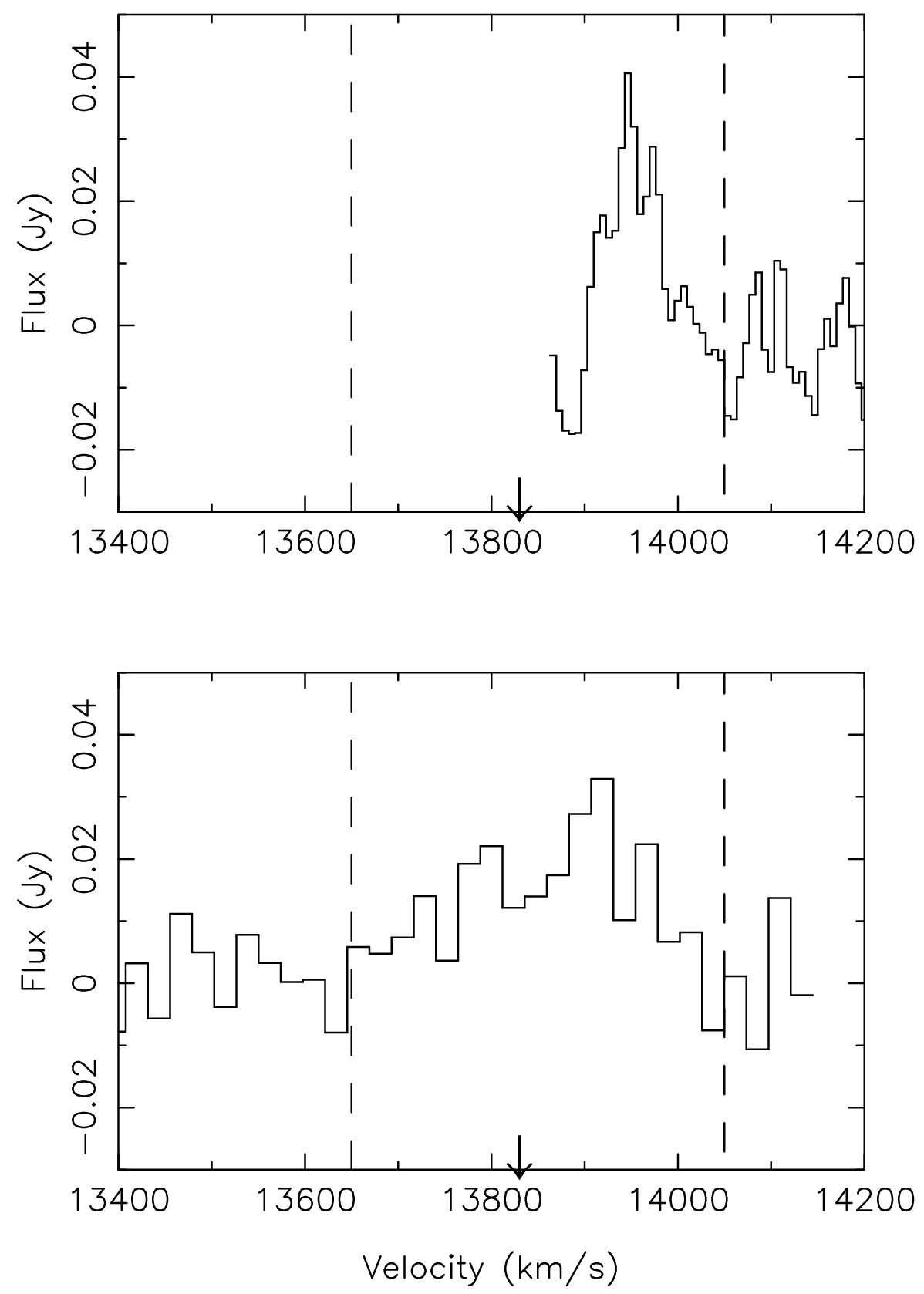


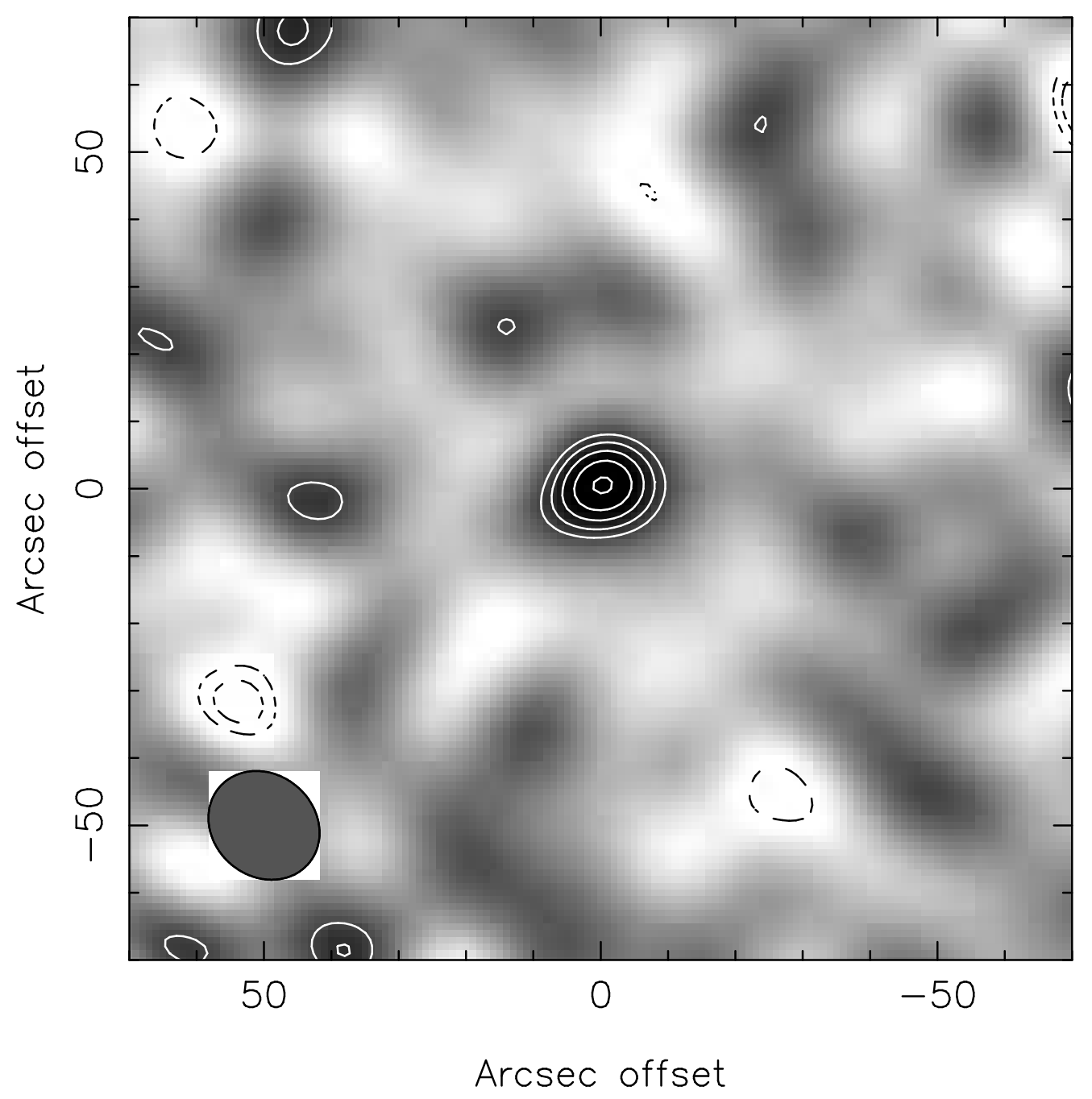

